\title{
An Empirical Analysis of Intel Thread Checker for Detecting Races in OpenMP Programs
}

\author{
Young-Joo Kim and Daeyoung Kim \\ School of Engineering \\ Information and Communications University \\ Daejeon, 305-732 South Korea \\ \{yjkim,kimd\}@icu.ac.kr
}

\author{
Yong-Kee Jun \\ Dep't of Information Science \\ Gyeongsang National University \\ Jinju, 660-701 South Korea \\ jun@gnu.ac.kr
}

\begin{abstract}
Races in OpenMP programs must be detected because they may cause unintended nondeterministic results of programs. The Intel Thread Checker can detect the races occurred in an execution of OpenMP program, but it has not been analyzed on the limitation of its power for race detection in OpenMP programs. This paper empirically analyzes the functionality and performance of Thread Checker using a set of multithreaded synthetic programs. We have found that Thread Checker can not verify the existence of races in an execution of OpenMP program, and show that the ratio of the required time for race detection is dependent on the number of total accesses to shared variables in parallel section programs, but not in parallel loop programs.
\end{abstract}

\section{Introduction}

Races $[8,11]$ in multithreaded programs occur when two parallel threads access a shared variable with at least a write access without proper inter-thread coordination. Especially, unintended races must be detected to debug programs because they may cause unintended nondeterministic results. Thread Checker $[1,2,10]$ dynamically detects such the races that occur in an execution of OpenMP programs using its projection technology. This technology checks the data dependency of accesses to shared variables using sequentially traced information, and reports anti, flow, and output data dependency as races but input data dependency. But its working principle has not been analyzed so that we do not know the limitations of its functionality and performance related to race detection. Due to this, users are not easy to understand efficiently the causes of reported races and does not know what the sensitive factors are in the performance of race detection.

This paper empirically analyzes the functionality and performance of Thread Checker using a set of OpenMP synthetic programs which include two kinds [12] of multithreaded structures: parallel loop and parallel section. The functionality analysis determines if the tool can verify the existence of races, and the performance analysis measures the required time, memory, and file-space for race detection. Our experimentation environment of Thread Checker is a multiprocessor system with an Intel 64-bit Zeon Dual processor and 1GB memory. With this system, the result of our analysis is as follows:

1. Functionality: Thread Checker can guarantees to verify the existence of races $[4,7,11]$ only in the programs without nested parallelism and interthread coordination.

2. Performance: Thread Checker shows that the ratio of the required time to the original time is dependent on the number of total accesses to shared variables in parallel section programs, but not in parallel loop programs.

Section 2 illustrates such the races that occur in OpenMP programs with directives, and indicates the problems that programmers have with Thread Checker. Section 3 presents our synthetic programs to experiment the functionality and performance of Thread Checker. Section 4 shows the results produced by the experiments and analyzes it with regard to the functionality and performance. The last section concludes our work and explores possible future work.

\section{Background}

This section illustrates the races which occur in an execution of OpenMP programs and introduces the problems that programmers have with Thread Checker for detecting races. We describe the races using an example program and a directed acyclic graph called POEG (Partial Order Execution Graph) [4].

\subsection{OpenMP Program}

OpenMP $[3,9]$ is an industry standard model of shared memory with a set of directives and libraries that sup- 


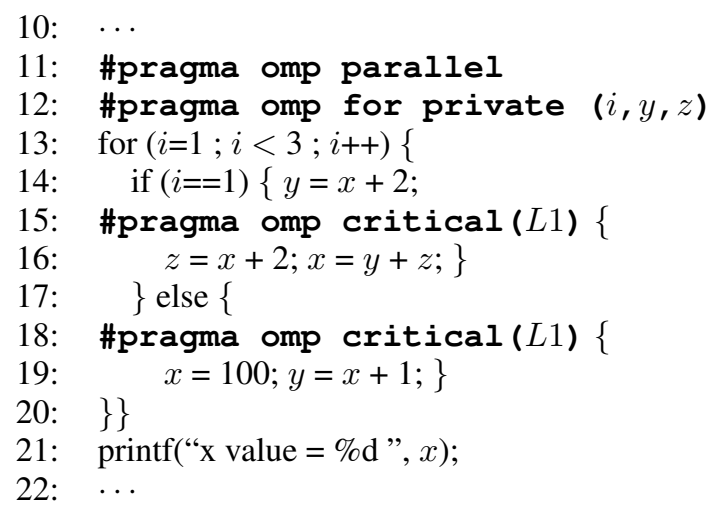

Figure 1. An OpenMP Program

port standard $\mathrm{C} / \mathrm{C}++$ and Fortran 77/90. OpenMP has parallelism directives and synchronization directives. Parallelism directives include "\#pragma omp parallel for" for parallel loop and "\#pragma omp section" for parallel section. If there is no other loop contained in a loop body, it is called the innermost loop. Otherwise, it is called an outer loop. In a nested loop, an individual loop can be enclosed by many outer loops. The nesting level of an individual loop is equal to one plus the number of the enclosing outer loops. The nesting depth of a loop is the maximum nesting level of loops in the loop. Synchronization directives include "\#pragma omp atomic," "\#pragma omp barrier," and "\#pragma omp critical" that control an execution order among threads. OpenMP also provides library functions and environment variables that can control run-time execution of programs.

In line 11 of Figure 1, two logical threads are created by "\#pragma omp parellel", and the integer variable $x$ is a shared variable shared by the two threads. During its executions, races may occur. We assume that variable $x$ has zero as an initial value, and the statements on line 14, 15, and 16 are executed by the first thread, and the statements on line 18 and 19 are executed by the second thread. These threads are logically concurrent with each other. Any unintended race does not exist toward variable $x$ between line 16 and line 19, because these two instruction blocks are protected from each other as critical sections by "\#pragma omp critical $(L 1)$." However, regarding the read access in the statement on line 14 and the write access to a shared variable $x$ in the statement on line 19, because the execution speed of the threads can be changed, race may occur between two accesses. Then the value of variable $x$ in the statement of line 21 may become 100 or 204 of intended value, but 104 of unintended value. this value occurs nondeterministically in programs execution, because these parallel threads are involved in a race toward the shared variable $x$.

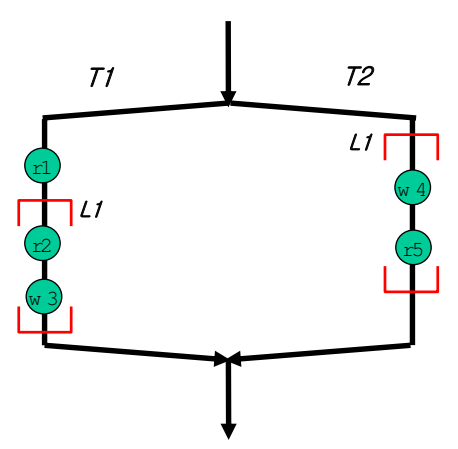

\section{Figure 2. OpenMP Program to express in POEG}

\section{$2.2 \quad$ Thread Checker}

Figure 2 represents an execution instance of Figure 1 by means of POEG [4]. A vertex of POEG means a fork or join operation for parallel threads, and an arc started from a vertex represents a thread started from the vertex. The accesses denoted $r$ and $w$ drawn with small disks upon the arcs identify read and write accesses which access a shared variable. A number attached to each access identifier indicates an observed order, and an arc segment delimited by the symbols $\Pi$ and $\sqcup$ means a critical section protected by a lock variable $L 1$. With POEG, we can easily understand partial order or happened-before relation [6] of accesses that occurred in an execution instance of programs. Figure 2 shows that $r 1$ and $w 4$ are involved in a race, because $r 1$ in thread $T 1$ and $w 4$ in thread $T 2$ are logically concurrent with each other, and $r 1$ is not protected by any lock variable.

Thread Checker $[2,10]$ of Intel Corporation can detect errors such as races in OpenMP programs with directives. The errors detected by Thread Checker are deadlock, race, and other logical errors. Intel $\mathrm{C} / \mathrm{C}++$ Compiler [5] instruments OpenMP programs. While the instrumented programs are executed serially, Thread Checker monitors the programs using its projection technology. This technology is applied only to the relaxed OpenMP programs which is parallelized only with OpenMP directives. Thread Checker detects races as follows. First, when such programs are compiled, a part of this tool integrated in the compiler modifies the programs to trace the information related to OpenMP directives and shared variables into an exclusive database. Second, when the complied program is executed sequentially, the tool uses the traced information in the database to check data dependency of accesses to shared variables whenever an OpenMP directive is located. Last, the tool reports the accesses as races if it satisfies an anti, flow, or output data dependency except input data dependency.

But about OpenMP programs, the tool's working principle has not been analyzed by now, so we do not know the 
limitations of its functionality and performance related to race detection. Due to this, users are not easy to understand efficiently the causes of the reported races and to know what the sensitive factors are concerning the performance of race detection.

\section{Experimentation}

This section presents our methodology to experiment the features of Thread Checker for two types of multithreaded program models [12] using synthetic programs regarding critical section, nesting depth, maximum parallelism, and the number of accesses.

\subsection{Criteria of Synthetic Programs}

An OpenMP program with directives may be described with either one of two types of multithreaded program models [12]: parallel loop programs for the parallel computing program model, and the parallel section programs for the activity management program model. The parallel loop program divides a single computation job into several parallel jobs; these jobs have the same kind of data structures and variables. The parallel section program creates a set of parent and child threads that have the allocated jobs often with different kinds of data structures which may be shared. Using these kinds of synthetic programs, we measure the accuracy and efficiency of Intel Thread Checker to analyze its functionality and performance.

To measure the accuracy, we observe whether the tool can verify the existence of races, and try to know the tool's principle of detecting races because its access maintenance policy can be comprehended. So we need to synthetic programs that consist of critical sections, nesting depth, and maximum parallelism. These synthetic programs have at most two accesses per thread. To measure the efficiency, we measure the time, memory, and file space required in race detection of the programs, and analyze the measured results for detection performance. So we need to synthetic programs that are defined with regard to the number of lock variable, the nesting depth, maximum parallelism, the number of accesses per thread, and the number of total accesses.

\subsection{Models of Synthetic Programs}

We classify synthetic programs for analyzing the tool's accuracy into sixteen program models using the four criteria: at most one lock variable, the nesting depth of one or two, the maximum parallelism of two or three, and the number of total accesses. Each synthetic program totally has three or four accesses which are distributed to one or two accesses in each thread of synthetic programs to understand the maintenance policy of accesses for race detection. The synthetic programs are named according to the usage identifier, the number of lock variables, nesting depth, and maximum parallelism.
For example, consider synthetic program "A-012-3" of Figure 3. "A" indicates the program is for the accuracy analysis; the first " 0 " means the number of lock variables to set critical sections; the second " 1 " indicates the nesting depth; the following " 2 " indicates the maximum parallelism; and the last " 3 " mean the number of total accesses. Figure 3 shows the POEG for the A-012-3 program and the observed accesses of the A-012-3 program where every number attached to each access indicates an observed order. We can analyze the maintenance policy of accesses from the patterns of races in Figure 3-(B). We classify synthetic programs for analyzing the tool's efficiency into two classes of models using the five criteria: the number of lock variables, the nesting depth, maximum parallelism, the number of accesses per thread, and the number of total accesses. One class is based on the number of accesses per thread, the maximum parallelism, and the nesting depth. The number of accesses per thread has four cases: 100, 200, 300 and 400. The maximum parallelism has five cases: 2, 4, 8, 16, and 32. The nesting depth has three cases: 0,1 , and 2. The other class is based on the number of total accesses, the maximum parallelism, and the number of lock variables. The number of total accesses has six cases: 1000, 2000, $3000,4000,5000$, and 6000. The maximum parallelism has five cases: $2,4,8,16$, and 32 . The number of critical sections per thread has three cases: 0,4 , and 8 . The synthetic programs are named differently according to two classes of the models.

For example, "P-02-1-100" is a synthetic program according to the number of accesses per thread. In the program, "P" indicates the program is for efficiency analysis, "02" means maximum parallelism, " 1 " indicates the nesting depth, and "100" means the number of accesses per thread. So the number of total accesses is 200. And "P-1000-020 " is a synthetic program according to the maximum parallelism. "1000" means the total number of accesses in the synthetic program, "02" means the maximum parallelism, and " 0 " means the number of lock variables. The number of accesses per this synthetic program is 500. Read ac-

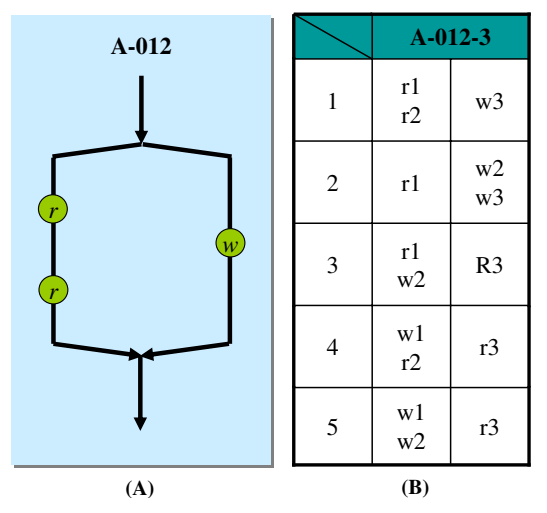

Figure 3. Synthetic Program A-012-3 

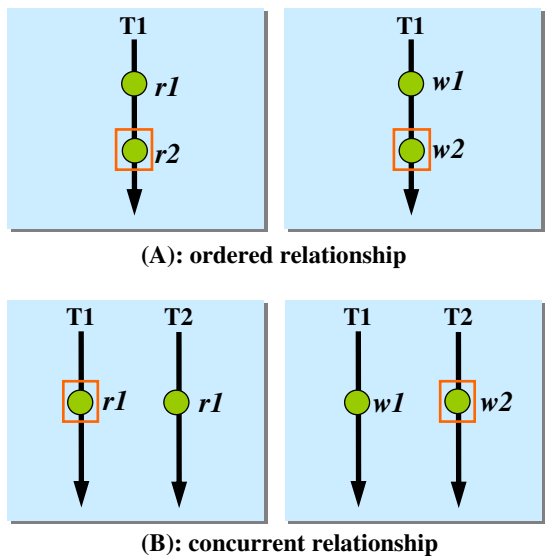

Figure 4. An Access's Management Policy: No Sync. and Non Nested Program Model

cesses only occur in odd threads and write accesses only occur in even threads. Efficiency can be analyzed according to the number of accesses and the maximum parallelism increment.

\section{Analysis}

The section analyzes the accuracy and efficiency of Thread Checker based on the results of experimentation using synthetic programs mentioned in section 3 on the Linux platform with Intel 64bit Zeon Dual processors.

\subsection{The Detection Principle}

We can understand the working principle of Thread Checker by analyzing the patterns of races detected in synthetic programs written for accuracy analysis. To understand the tool's working principle, we must grasp the maintenance policy of accesses. So we check ordered or concurrent relation for all accesses which occur in a program execution and then comprehend whether the accesses are stored in a storage such as exclusive database. The policy is as follows. If the current read access $r_{j}$ is ordered with the previous read access $r_{i}$, the $r_{j}$ is stored and $r_{i}$ is deleted in the storage. Also, if the current write access $w_{j}$ is ordered with the previous write access $w_{i}$, the $w_{j}$ is stored and $w_{i}$ is deleted in the storage.

For example, Figure 4 shows the maintenance policy of read accesses and write accesses in the program without synchronization and nested parallelism. If present read access $r 2$ and previous read access $r 1$ are ordered relation in Figure 4-(A), $r 2$ is stored and $r 1$ is deleted. And if present write access $w 2$ and previous write access $w 1$ are ordered relation, $w 2$ is stored and $w 1$ is deleted. If present read access $r 2$ and previous write access $r 1$ are concurrent relation

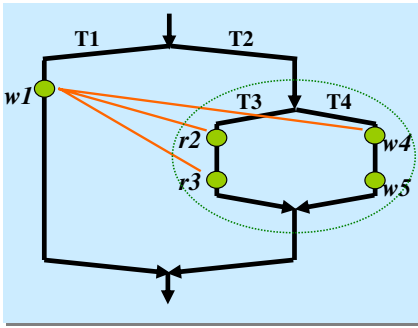

(A)

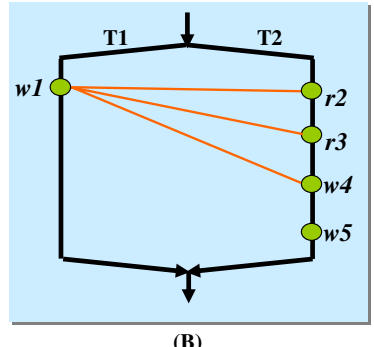

Figure 5. An Access's Maintenance Policy: No Sync. and Nested Program Model in Figure 4-(B), $r 1$ is stored and $r 2$ is deleted. And present write access $w 2$ and previous write access $w 1$ are in parallel, $w 2$ is stored and $w 1$ is deleted. Figure 5 shows an access's maintenance policy of Thread Checker in the program without synchronization and with nested parallelism. Figure 5-(A) and (B) are different program models but they have the same access's maintenance policy of accesses. Because Thread Checker regards nested sibling threads as ordered threads in the program with nested parallelism. For example, in Figure 5-(A), the reason why races are not reported in $r 2, r 3, w 4$, and $w 5$ is because thread $T 3$ and $T 4$ are sibling threads. Also the reason why $w 1$ and $w 5$ are not reported as races is because when $w 4$ happened, the $w 4$ is concurrent with the $w 1$ and so the $w 1$ is deleted in shared data structure. And if nested thread T3 and T4 in Figure 5(A) are set as the orphan directive, Thread Checker does not report races.

\subsection{Accuracy Analysis}

Figure 6 and Figure 7 are to show the detected races in "A-012-3," "A-012-4," and "A-112-3." Figure 6-(A), Figure 7-(A), and Figure 7-(B) have three accesses and Figure 6-(B) has four accesses. In four synthetic programs which generate two threads, a number attached to each access indicates an occurrence order of accesses and symbol [ ] indicates a critical section. Figure 6 shows that Thread Checker can verify the existence of races because of reporting at least one race. For example, the first program of Figure 6-(A) has one race: " $r 2-w 3$." We can understand the detection result of Figure 6 by using the detection principle mentioned in section 4.1. But Figure 7 shows that Thread Checker cannot verify the existence of race because of not reporting at least one race. For example, the second program of Figure 7-(A) has one race: " $r 1-w 3$." Thread Checker does not report this race. The first program of Figure 7-(A) does not have races because accesses are protected by critical section. Therefore, it is impossible to verify the existence of races in the program with synchronization and non nested parallelism. As we summarize the 


\begin{tabular}{|c|c|c|c|}
\hline & \multicolumn{2}{|c|}{ A-012-3 } & report \\
\hline 1 & $\begin{array}{c}\text { r1 } \\
\text { r2 }\end{array}$ & w3 & r2-w3 \\
\hline 2 & r1 & $\begin{array}{c}\text { w2 } \\
\text { w3 }\end{array}$ & $\begin{array}{l}\text { r1-w2 } \\
\text { r1-w3 }\end{array}$ \\
\hline 3 & r1 & r3 & w2-r3 \\
\hline 4 & w1 & r3 & w1-r3 \\
\hline 5 & w1 & r3 & w2-r3 \\
\hline
\end{tabular}

(A)

\begin{tabular}{|c|c|c|c|}
\hline & \multicolumn{2}{|c|}{ A-012-4 } & report \\
\hline 1 & $\begin{array}{c}\text { r1 } \\
\text { r2 }\end{array}$ & $\begin{array}{c}\text { w3 } \\
\text { w4 }\end{array}$ & $\begin{array}{c}\text { r2-w3 } \\
\text { r2-w4 }\end{array}$ \\
\hline 2 & $\begin{array}{c}\text { r1 } \\
\text { w2 }\end{array}$ & $\begin{array}{c}\text { r3 } \\
\text { w4 }\end{array}$ & $\begin{array}{c}\text { w2-r3 } \\
\text { r1-w4 } \\
\text { w2-w4 }\end{array}$ \\
\hline 3 & $\begin{array}{c}\text { w1 } \\
\text { r2 }\end{array}$ & $\begin{array}{c}\text { r3 } \\
\text { w }\end{array}$ & $\begin{array}{c}\text { w1-r3 } \\
\text { r2-w4 } \\
\text { w1-w4 }\end{array}$ \\
\hline 4 & $\begin{array}{c}\text { r1 } \\
\text { w2 }\end{array}$ & $\begin{array}{c}\text { w3 } \\
\text { r4 }\end{array}$ & $\begin{array}{c}\text { r1-w3 } \\
\text { w2-w3 }\end{array}$ \\
\hline 5 & $\begin{array}{c}\text { w1 } \\
\text { r2 }\end{array}$ & $\begin{array}{c}\text { w3 } \\
\text { r4 }\end{array}$ & $\begin{array}{c}\text { w1-w3 } \\
\text { r2-w3 }\end{array}$ \\
\hline
\end{tabular}

(B)
Figure 6. The Result Detected in A-012-3 and A-012-4

accuracy analysis result with the synthetic programs mentioned in section 3, Thread Checker can verify the existence of races in "A-012-3" and "A-012-4," but can not verify the existence of races in "A-112-3" and "A-112-3." Also, thread Checker can not verify races in synthetic programs with nested parallelism because it regards nested sibling threads as ordered threads.

\subsection{Efficiency Analysis: Parallel Section Programs}

The tool's efficiency is analyzed using the synthetic programs mentioned in section 3. So we measure the time, memory, and file-space required for detecting races only in the synthetic programs which race verification is possible in the result of the accuracy analysis. And we must execute the synthetic programs in two modes to get an experimentation result: original mode is to execute the programs without monitoring for race detection and transformation mode is to execute the programs with monitoring for race detection.

In original mode of "P-16-0-400," the required time is
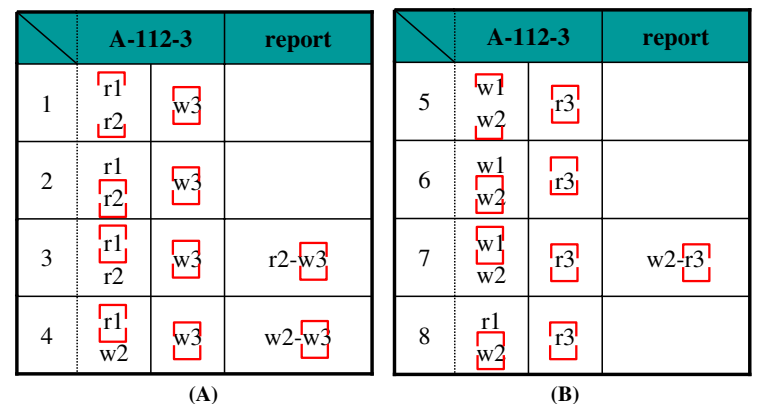

Figure 7. The Result Detected in A-112-3
$0.002 \mathrm{sec}$, the required memory can not be measured because the execution time is so short, and the required filespace is $14 \mathrm{~KB}$. In transformation mode of "P-16-0-400," the required time is $15.087 \mathrm{sec}$, the required memory is about $22 \mathrm{MB}$, and the required file-space is $589 \mathrm{~KB}$. As the maximum parallelism increases, the ratio of the required time, memory, and file-space becomes greater. In original mode of "P-6000-16-0," the required time is $0,002 \mathrm{sec}$, the required memory also can not be measured, and the required file-space is $13.53 \mathrm{~KB}$. In transformation mode of "P-6000$16-0$," the required time is $15.091 \mathrm{sec}$, the required memory is about $22 \mathrm{MB}$, and the required file-space is $546.96 \mathrm{~KB}$. As the number of total accesses increase, the ratio of the required time, memory, and file-space becomes also greater.

Figure 8 shows the average ratio of transformation mode about original mode in the aspect of the required time, memory, and file-space. These data are to experiment in two aspects of synthetic programs: according to the number of accesses per thread and the maximum parallelism. For accuracy of the measured data, we have verify the data using $99 \%$ Confidence Interval. In the synthetic programs according to maximum parallelism, when the number of total accesses is 1000, the required time increases 3769 times than original programs on average. And when the number of total accesses is 4000 , the ratio increases 11488 times on average. And when maximum parallelism is 16 , the ratio increases 6147 times on average. The ratio of the required memory and file-space is not greater than the ratio of the required time. If the number of accesses or the maximum parallelism are increase, the number of total accesses also increases. Therefore, we can know that the ratio of the required time depends on the number of total accesses

\subsection{Efficiency Analysis: Total Accesses}

We must comprehend whether the ratio of the required time in the multiprocessor depends on the number of total accesses. So we write the synthetic programs based on parallel loop programs and parallel section programs which the number of total accesses has six cases: 1000, 2000, 3000, 4000,5000 , and 6000 and the maximum parallelism has five cases: 2, 4, 8, 16, and 32. We experiment the synthetic programs in an Intel 64bit Zeon Dual processor system which is recognized as four processors. Figure 9 shows the results

\begin{tabular}{|c|c|c|c|c|c|c|}
\hline & \multicolumn{2}{|c|}{$\begin{array}{l}\text { \# Maximum Parallelism } \\
\text { (Times) } \\
\end{array}$} & \multicolumn{4}{|c|}{$\begin{array}{c}\text { \# Access per Thread } \\
\text { (Times) } \\
\end{array}$} \\
\hline & 1000 & 4000 & 2 & 4 & 8 & 16 \\
\hline Time & 3769 & 11488 & 2256 & 3383 & 6122 & 6147 \\
\hline Memory & 14 & 21 & 10 & 14 & 19 & 32 \\
\hline File-Space & 7 & 41 & 4 & 7 & 14 & 27 \\
\hline
\end{tabular}

Figure 8. Effiency Analysis 


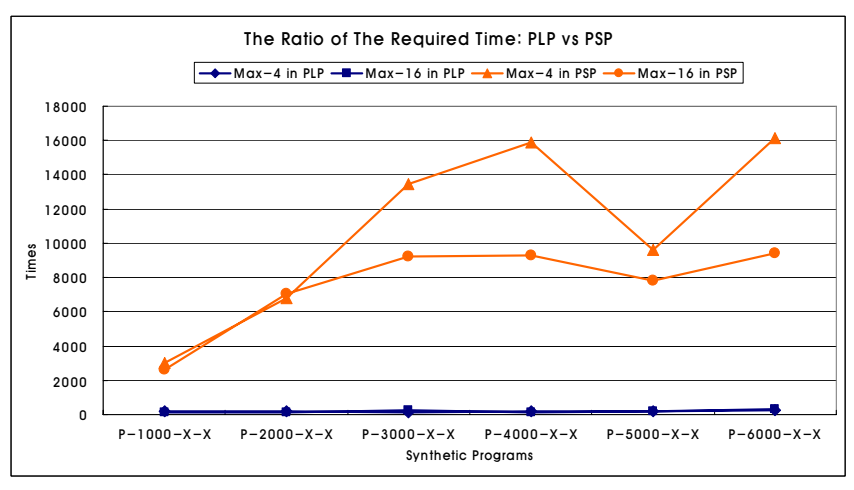

Figure 9. Experimentation Result

which measure the time required for race detection and the measured results satisfy the Confidence Index 99\%.

In Figure 9, the horizontal (x) axis indicates synthetic programs and the vertical (y) axis indicates the ratio of the required time. "Max-4 in PLP" means the ratio of the time required for detecting races in parallel loop program with maximum parallelism 4. "Max-4 in PSP" indicates the ratio of the time required for detecting races in parallel section program with maximum parallelism 4. And "P-1000X-X" has two means; "P-1000-4-0" and "P-1000-16-0." As we look into the experimentation result of Figure 9, we can know that the required time in parallel computing programs is independent of an incremental of the number of total accesses, because there is scarcely the ratio of the required time for "Max-4 in PLP" and "Max-16 in PLP." The required time for "Max-4 in PSP" and "Max-16 in PSP" increases proportionately as many as $O(n)$ where $n$ means the number of total accesses. Therefore, we can know empirically that Intel Thread Checker efficiently detects races in parallel loop programs but that it is an impractical tool in parallel section programs.

\section{Conclusion}

Thread Checker is the representative tool for detecting races during an execution of OpenMP program with directives. We could know empirically that Thread Checker can not verify the existence of races and that it efficiently detects races in parallel loop program. But it is an impractical tool in parallel section program. Through the analysis result of functionality and performance, we were able to grasp the working principle of Thread Checker. Understanding of the detected races will be increased if this working principle is used in race detection. This principle will help in the design of either a race detection tool with improved in the aspect of functionality and performance or an environment for effectively debugging. In the future work, the task remains to design a race detection tool with functionality and performance superior to that of Thread Checker.

\section{References}

[1] Banerjee, U., B. Bliss, Z. Ma, and P. Petersen, "A Theory of Data Race Detection." Proc. of Workshop on Parallel and Distributed Systems: Testing and Debugging (PADTAD), pp. 69-78, ACM, Portland, USA, July 2006.

[2] Banerjee, U., B. Bliss, Z. Ma, and P. Petersen, "Unraveling Data Race Detection in the Intel Thread Checker." Proc. of Workshop on Software Tools for Multi-core Systems (STMCS06), Manhattan, New York, NY., USA, March 2006.

[3] Dagum, L., and R. Menon, "OpenMP: An IndustryStandard API for Shared Memory Programming," Computational Science and Engineering, 5(1): 46-55, IEEE, January-March 1998.

[4] Dinning, A., and E. Schonberg, "An Empirical Comparison of Monitoring Algorithms for Access Anomaly Detection," 2nd Symp. on Principles and Practice of Parallel Programming, ACM, pp. 1-10, March 1990.

[5] Intel Co., Intel C++ Compiler 8.1 for Windows: Getting Started Guide, 2004.

[6] Lamport, L., "Time, Clocks, and the Ordering of Events in a Distributed System," Comm. of ACM, 21(7): 558565, July 1978.

[7] Mellor-Crummey, J. M., "On-the-fly Detection of Data Races for Programs with Nested Fork-Join Parallelism," Supercomputing, pp. 24-33, ACM/IEEE, Nov. 1991.

[8] Netzer, R. H. B., and B. P. Miller, "What Are Race Conditions? Some Issues and Formalizations, Letters on Prog. Lang. and Systems, 1(1): 74-88, ACM, March 1992.

[9] OpenMP Architecture Review Board, OpenMP Application Programs Interface, Version 2.5, May 2005.

[10] Petersen, P., and S. Shah, "OpenMP Support in the Intel Thread Checker," Int'l Workshop on OpenMP Applications and Tools (WOMPAT), pp. 1-12, June 2003.

[11] Park, S., M. Park, and Y. Jun, "A Comparision of Scalable Labeling Schemes for Detecting Races in OpenMP Programs," Int'l Workshop on OpenMP Applications and Tools (WOMPAT), pp. 66-80, West lafayette, Indiana, July 2001.

[12] Rinard. M., "Analysis of Multithreaded Programs," Int'l Static Analysis Symposium (SAS), Lecture Notes in Computer Science, 2126: 1-19, Springer-Verlag, July 2001. 